\title{
Localization method based on omnidirectional stereoscopic vision and dead-reckoning
}

\author{
Cyril DROCOURT, Laurent DELAHOCHE, Claude PEGARD, Cyril CAUCHOIS \\ GRACSY - 7 rue du Moulin Neuf, 80000 Amiens - FRANCE \\ Cyril.Drocourt@iut.u-picardie.fr , Laurent.Delahoche@u-picardie.fr
}

\begin{abstract}
This paper presents a system of absolute localization based on the cooperation of a stereoscopic omnidirectional vision system and a dead-reckoning system. To do it we use an original perception system which allows our omnidirectional vision sensor SYCLOP to move along a rail. The first part of our study will deal with the problem of building the sensorial model with the help of the two stereoscopic omnidirectional images. To solve this problem we propose an approach based on the fusion of several criteria which will be made according to Dempster-Shafer rules. As for the second part, it will be devoted to exploiting this sensorial model to localize the robot thanks to matching the sensorial primitives with the environment map. We use the dead-reckoning prediction to decrease the combinatory aspect of the matching algorithm. We analyze the performance of our global absolute localization system on several robot's elementary moves, in an indoor environment.
\end{abstract}

\section{I.Introduction}

The localization problem is very important for the navigation paradigm. Then, localizing the robot in its evolution environment constitutes a prerequisite step to any decision making [4].

Two kinds of localization method can be distinguished : the absolute localization methods and the relative localization methods. Localization methods which can satisfy the constraints imposed by the navigation, are generally based on locating artificial beacons. This kind of system is generally employed for industrial applications as for example the navigation system developed by DurrantWhyte [6] for an Autonomous Guided Vehicle which transports containers. These methods are fast and reliable, but unfortunately they lack flexibility and modularity because it is necessary to fit out the robot's evolution environment.

Another category of method consists in referencing directly on characteristic elements of the robot's evolution environment. Indeed, these solutions offer a great modularity and allow the robot to localize itself in accordance to the landmarks. This kind of localiz ation is generally founded on a matching stage between a sensorial model and a theoretic map of the environment. The perception systems used in that case are often the vision systems and the range finding ones. Thus, Gonzalez in [7] determines the absolute position of its robot by using the line segments as sensorial primitives. These are obtained thanks to a rotating laser rangefinder. In [4], Leonard develops a method of dynamic localization based on the location of "geometric beacons", which are detected by a belt of ultrasonic sensors. These geometric beacons are determined thanks to regions of constant depth (RCD). Atiya [2], use a CCD camera to detect vertical lines of the environment as natural beacons. Similarly Yagi uses an omnidirectional vision system to develop navigation and environment map building methods [3]. We can notice that the robustness of this kind of localization methods is mainly linked to the matching stage. The more precise and rich information the sensorial model will give, the more robust the matching stage will be. That is why we have worked on an original method of sensorial model building based on the use of a stereoscopic omnidirectional perception system. In order to reduce the high combinatory aspect of the matching stage and to increase the robustness of this stage, we use a position estimation given by an odometer.

The first part of this paper presents the principle of our stereoscopic omnidirectional perception system. The second part will deal with our sensorial model building method using the multicriteria fusion, made according to Dempster-Shafer rules. Our absolute localization method will be presented in the last part. In the conclusion we will analyze the experimental results reached with our mobile robot SARAH.

\section{The stereoscopic omnidirectional sensor}

The stereoscopic omnidirectional sensor put on our mobile robot SARAH is based on the rigid translation of the omnidirectional vision system SYCLOP used in our laboratory [5]. The SYCLOP system is similar to the COPIS one used by YAGI [3] and is composed of a conic mirror and a CCD camera (Figure 1). It allows us to detect all the vertical landmarks of the environment thanks to a dimensional projection.

The rigid translation has been made thanks to two horizontal rails which allow a precise straight move in the horizontal SYCLOP sensor plan. Thus the system insures 
the acquisition of two omnidirectional images of the environment within 40 centimeters of one another. The distance between these two shots has been determined experimentally by making a compromise between congestion and precision in the sensorial primitive determination.

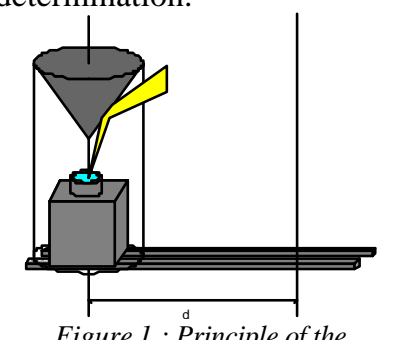

stereoscopic omnidirectional sensor

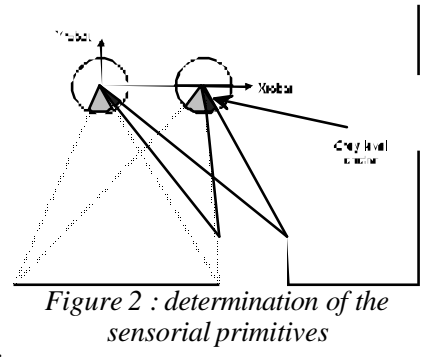

If for each point we know the two radial straight lines generated on each cone (Figure 2), we can calculate the position of the vertical landmarks (edges, corners,

Three treatments are necessary to get these points :

- the radial segment of the two images

- the matching of the sector thus found

- the calculation of the coordinates of the points found

\section{III.Sensorial primitive treatments}

We want to treat the radial straight lines corresponding to the vertical landmarks. The lines being all radial and generally distributed homogeneously on 360 degrees, we take into consideration five concentric grey level circles whose average is then made. We obtain thus one grey level curve for each image (Figure 3).

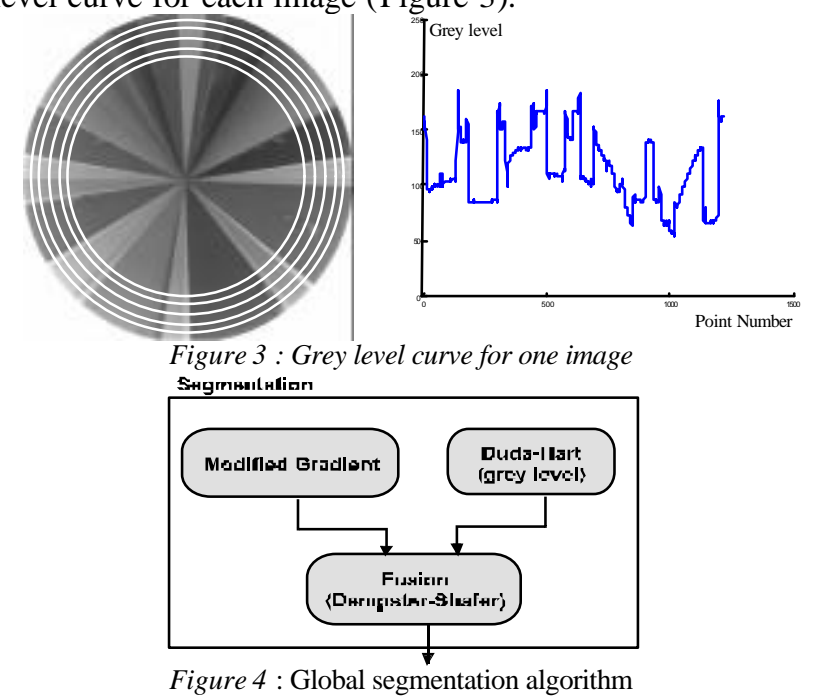

It is then necessary to treat this signal of grey level to extract the great variation which corresponds to sector changes. We propose a segmentation method based on the fusion of two complementary treatments (Figure 4). The fusion is made thanks to the combination rules of the Dempster-Shafer theory [8].

\section{III.1 - Gradient segmentation method}

We apply a gradient vector on the grey level curve. To limit the noise importance we have decided to use a vector of dimension $7:\left[\begin{array}{lllllll}-2 & -2 & -1 & 0 & 1 & 2 & 2\end{array}\right]$. The problem of this classical approach is that a change of sector generates a multitude of radial straight lines. That's why we have developed a post-treatment to filter the gradient curve : on the curves we look for the local maximum and minimum and set all the other points at zero. During the thresholding, this treatment allows to take into consideration only the characteristic points in the sector building. In spite of a better detection of the radial straight lines, we can note the apparition of quite a big number of noisy straight lines. So, it seems necessary to plan another complementary treatment.

\section{III.2 - Duda-Hart segmentation method}

The Duda-Hart algorithm [9] is mainly used as a segmentation method on a set of points as in rangefinding data for example [1]. It consists in grouping recursively sets of lined up points in connection with distance criterion point-segment. The algorithm is stopped when there are no points left to check the distance condition. By applying this method to our grey level curves, we take into consideration the grey level continuity criterion (Figure 5). This criterion is complementary to the first one, based on the breaking between the grey level ranges.

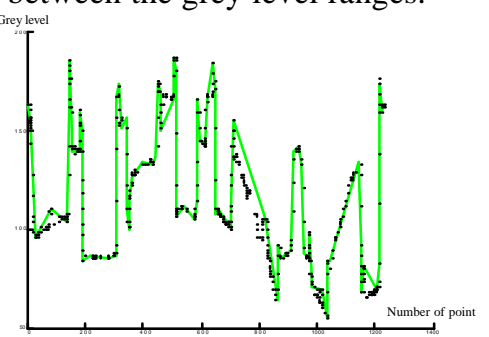

Figure 5 : Duda-Hart algorithm applied to a grey level curve

We must note a few points concerning this criterion :

- there isn't an important number of radial straight lines for a separation between two sectors.

- When a breaking point (radial straight line) is detected by this algorithm, its existence must be validated with the gradient criterion. On the contrary, if this algorithm has detected no straight line, there is a very great probability that none exists.

- The lighting conditions have little influence on the number of detected lines, contrary to the gradient (threshold problem).

The next step consists in merging these two complementary methods.

\section{III.3 - Segmentation data fusion}

The fusion of the previous methods consists in solving two problems : 
- Finding an automatic threshold method for the two treatments.

- Merging the two types of information.

Using the Dempster-Shafer rules [8] will allow us to solve these two problems. Then we consider the $f_{l}$ function corresponding to the absolute value of the gradient and the $f_{2}$ function corresponding to the Duda-Hart algorithm. For each point of the grey level curves, the $f_{2}$ function will be defined as equal to zero for the lined up points and equal to the distance breakpoint-segment which has created the division of a set of points into two segments.

We then try to calculate a minimum value under which no radial line can exist and a maximum value above which a straight line can exist. The further the value of a point moves from the average of all the points, the more probable it is to be a sector separation according to Dempster-Shafer. As the standard deviation represents the average deviation in relation to the average, we have decided to center the two curves of the value "Average+standard deviation" and to work on the following interval : [Average; Average $+(2 \times$ standard deviation $)]$

Then we reduce this interval to $[0 ; 1]$ so that all the points from the two curves could be represented in this "ambiguity window". We have given the value 0 to all the points situated below this interval and the value 1 for all the points above. Our frame of discernment is thus composed of two elements : "YES" and "NO" corresponding to those assertions :

- "yes, a radial straight line exists"

- "no, a radial straight line doesn't exist"

So, we can write the following 4 Basic Probability Assignment (B.P.A.) :

$$
\begin{array}{ll}
m_{1}(Y E S)=f_{1} & M_{2}(Y E S)=f_{2} \\
m_{1}(N O)=1-f_{l} & m_{2}(N O)=1-f_{2}
\end{array}
$$

We can then perform the combination calculation thanks to the Dempster-Shafer rules [8] which now allows to calculate the conflict coefficient between our two elements of the frame of discernment :

$k=m_{1}(Y E S) \cdot m_{2}(N O)+m_{1}(N O) \cdot m_{2}(Y E S)$

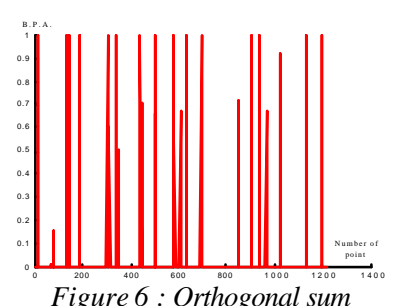

Figure 6: Orthogonal sum $m 1 \oplus n 2(O U I)$

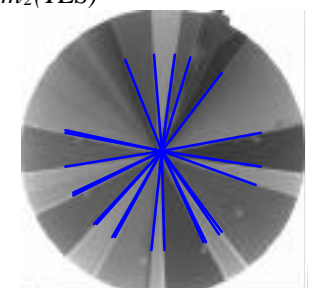

Figure 7 : Segmentation obtained with the fusion

If $\mathrm{k}<1$ the conflict is not complete and the combination of belief functions (figure 6) for each $\beta$ element of the frame of discernment is given by (Figure 6):

$$
m_{1} \otimes m_{2}(\beta)=\frac{m_{1}(\beta) \cdot m_{2}(\beta)}{1-k}
$$

To determine the existence of radial straight lines (Figure 7), we take all the points whose B.P.A. for the YES is superior to the $N O$.

\section{Sector matching algorithm}

The matching stage consists in matching the different grey level sectors detected in the two images with a maximum of robustness. Here again we use DempsterShafer for the fusion of the different criteria (Figure 8).

Because the two cones of the perception system are far from one another, the vertical landmarks cannot appear identically on the two images. Indeed some sectors can appear on one image but not on the other because of occultation problems or because of the difference of the two segmentations. Thus the sectors will not follow one another in the same order on the two images. It is thus necessary to find more significant matching criteria than the order of apparition. To solve this problem we have selected 4 comparison criteria for each sector :

- The inclination of the approximate straight lines of the set of sector points.

- The average of the grey level sector.

- The standard deviation of the grey level sectors.

- The geometrical criterion of the sector.

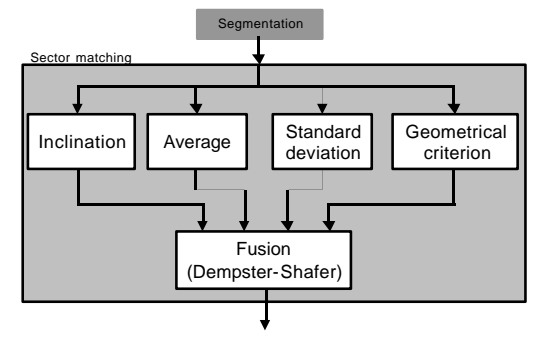

Figure 8 : Global matching sector algorithm

\section{IV.1 - Matching criteria determination}

The first matching criterion is calculated from the grey level curves. It consists in approximating the set of points corresponding to each sector thanks to a least square line. We have noted that the orientation of this line segment (Duda-Hart segmentation of the Figure 5) varied little between the two images for a given sector : this constitutes experimentally a good matching criterion.

The second chosen criterion is the average of the sector taken on the grey level curve.

The third one is the standard deviation of each sector also taken on this curve. It is complementary to the second one as it represents the dispersion around the average.

The last criterion is the geometrical one. We consider the plan containing the base of the two cones $A$ and $B$ with a radius $R$, separated by the distance $d$. We suppose this plan to be parallel to the one on which the robot moves. We consider that the robot's center of reference is situated on the center of the cone A and that the two cones are lined on the axis Ox (Figure 10).

Two cases can be contemplated : the considered landmark is detected in the same circle quarter on the two images but with two different angles, or in adjacent 
symmetrical circle quarters (Figure 10). Indeed a vertical landmark seen of the right by the left sensor is obligatorily seen on the right by the right sensor. Moreover the angle made each side of the sector on the left cone must be superior to the angle of each side corresponding to this sector on the right cone (Figure 10).

\section{IV.2 - Matching criteria fusion}

Our fusion method used the Dempster-Shafer's rules. The matching of the sector of an image with another sector must be managed with a positive and negative answer. That's why our discernment frame is made of two elements "YES" for "yes the two sectors must be matched" and "NO" for "no the two sectors must not be matched". By considering four sensors corresponding to the four matching criteria, we can establish the B.P.A. of each sensor for the two elements of the frame of discernment. To know if the $\gamma$ sector of an image must be matched with the sector $\lambda$ of the other image (Figure 10), we must set up the matching function between the output of our sensors and the B.P.A. of each element in the frame of discernment (Figure 9).

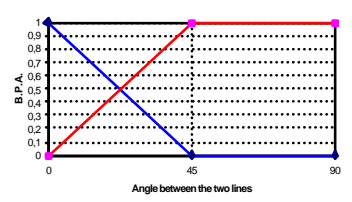

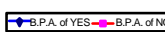

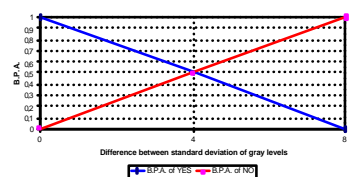

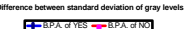
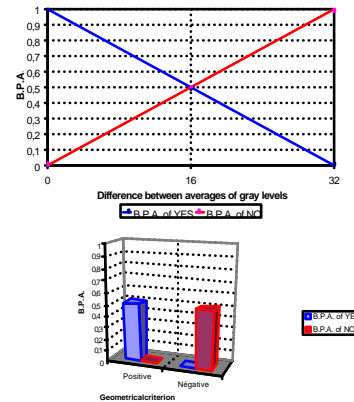

Figure 9 : Matching functions for the sector matching stage

The first three functions correspond to the difference of the first three sensors between the $\gamma$ and the $\lambda$ sector. The last function is given by the geometrical criterion :

- If the $\gamma$ sector can be geometrically matched to the $\lambda$ sector, the B.P.A. for " $Y E S$ " is equal to 0.5 ,

- $\quad$ otherwise the discernment frame is equal to 0 .

We can then calculate the B.P.A. and the $k$ conflict coefficient between the two elements of the frame of discernment. If $\mathrm{k}<1$ then the conflict is not complete and the combinion of belief functions for each $\beta$ of the frame of discernment is given by :

$$
m_{1} \otimes m_{2} \otimes m_{3} \otimes m_{4}(\beta)=\frac{m_{1}(\beta) \cdot m_{2}(\beta) \cdot m_{3}(\beta) \cdot m_{4}(\beta)}{1-k}
$$

\section{IV.3 - Global matching algorithm}

We know that the geometrical criterion reduces the research of the sectors which can be matched, but also that a sector can be matched only once. That's why the global matching algorithm is composed of three treatments (Figure 10) :
- First, we calculate the matching of the sectors which have the highest geometric criterion : those of the A-I zone. They must then be matched with the sectors of the B-I zone.

- Secondly, we try to match those of the B-II zone with the sectors of the A-II zone.

- Lastly, the sectors of the A-II zone must be matched with the remaining ones of the B-I zone.

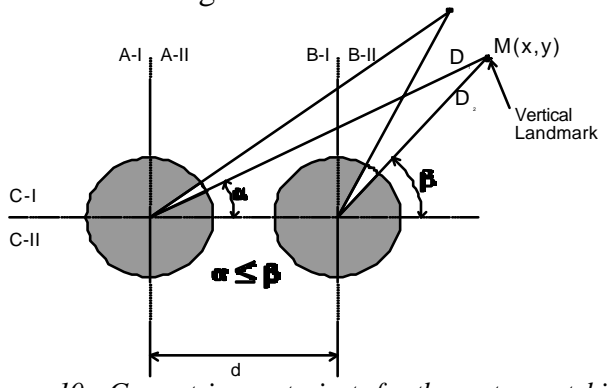

Figure 10: Geometric constrainsts for the sector matching
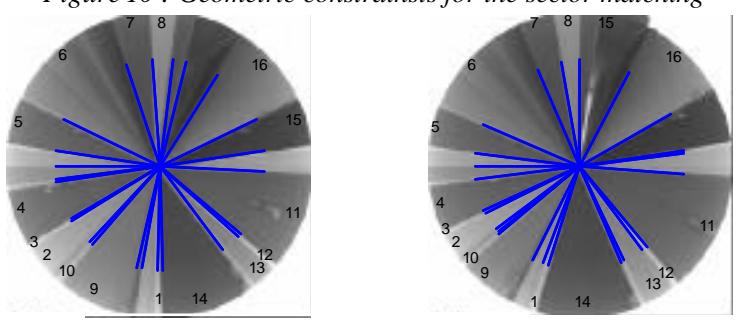

Figure 11 : Segmentation and final sector matching for the two images corresponding to a stereoscopic acquisition

In order not to commit matching errors, we keep a solution only if it is unique, otherwise no decision is taken. In that case the algorithm continues with the other sectors. What we call the unique solution is here the maximum solution superior to the probability 0.5 and distant from another point of at least 0.1 . For each matching, we repeat this stage. Indeed a matching can allow to remove previous matching ambiguities.

\section{Landmark position determination}

Once the sectors have been matched, we have only to calculate the points of the segments we represent. To do it, we know the orientation angle of straight lines defining a sector and the distance separating the two cones. The coordinates of all the points in sensor references are calculated with :

$$
x=\frac{d \times \tan (\beta)}{\tan (\beta)-\tan (\alpha)} \quad y=\frac{d \times \tan (\beta) \times \tan (\alpha)}{\tan (\beta)-\tan (\alpha)}
$$

\section{Localization algorithm}

We get a sensorial model where two types of sensorial primitives can be considered : the points which characterize vertical landmarks, or the segments characterizing a horizontal landmark.

We have two possibilities to localize the robot :

- Matching the points of the sensorial model with the 
points listed in the theoretical environment map,

- Matching the segments of the sensorial model with the theoretical map segments

Theoretically each couple of matched sectors represents a horizontal landmark. We can see that in some cases it is not checked. For example, the vacuum (door opening, corridor ...) is characterized by a segment. We can thus say that if we consider segments as sensorial data, more horizontal landmarks will appear. We can add another case where a couple of sector represents a set of horizontal landmarks. Finally for certain angular configurations an important error can appear on a segment orientation coefficient. The accumulation of all these errors can in some cases create a sensorial model which can't be exploited from the segment point of view for the matching algorithm.

That's why we have decided to consider more robust geometric primitives than the segments, namely the points.

The most robust convergence criterion that we have kept for the matching algorithm is based on the Cartesian distance between each sensorial model point and the nearest one of the environment map.

By superposing the sensorial model experimentally got with the environment map we can notice a relatively important precision in the determination of the points coordinates (Figure 13). So, we use this superposition directly to localize the robot from the translation and the rotation obtained with the matching algorithm. The principle of our localization method is finally as follows :

- we consider 2 points of the sensorial model

- we look for 2 points of the theoretical model corresponding to the 2 chosen points in connection with the Cartesian distance.

- we calculate the distance separating the sensorial model remaining points from the nearest theoretically model points. This constitutes our final selection criterion.

The first stage of this algorithm is strongly combinatorial. The number of possible couples of points is equal to $n(n-1) / 2$ for $n$ points of the sensorial model.

The first amelioration to reduce this complexity consists in using only the two points from the matching of an identical sector, which permits to reduce the number of possible couples to $n$.

To find the theoretical model couple which best corresponds to the sensorial model, we must calculate the distance which separates these points. We then compare the two distances (sensorial and theoretical) for all the couples of points of the theoretical model. Once a solution has been found, we calculate the rotating angle which must be applied to the set of remaining points.

About this comparison stage we can note that smaller the number of tested segments matching combinations is, the more robust the matching stage is. Moreover in order to reduce the complexity of this stage, the reduction of tested couples of points combination is necessary. For this, we use the position estimation and its associated incertitude domain given by dead-reckoning. We obtain the position and the incertitude domain with the classical equations linked to the dead-reckoning [10] [5]. In our matching problem, we determine a domain of possible absolute position from the incertitude ellipse which we assimilate as a circle. The radius of these circles are calculated with the length of the major axis of the incertitude ellipse. Then the second amelioration of the initial matching algorithm consists in ejecting the combinations of sensorial points couples and theoretical points which generate a position out of the domain of possible positions. This couples selection stage permits to avoid computing the (n-2) points association after the couple of points matching.

Lastly we have to calculate, for each remaining sensorial model point, the minimal distance which separates it from a point of the theoretical model. The cumulated distance (sensorial point-theoretical point) allows to choose the best position. Our selection criterion of the final solution is then the minimum cumulated distance error.

\section{VII.Experimental results}

To test the robustness of our localization algorithm, we have executed it on several sensorial acquisitions made in an indoor environment (Figure 12). The acquisitions are made when the robot has stopped, with our stereoscopic omnidirectional sensor shown on Figure 12.

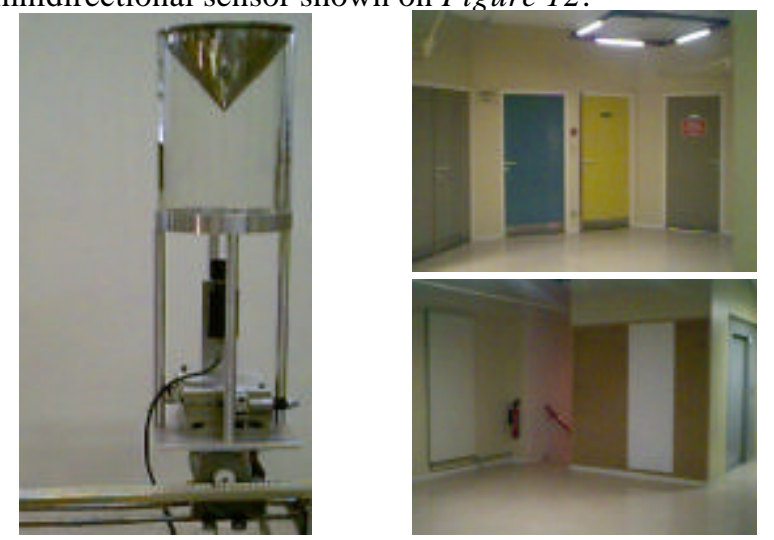

Figure 12 : Our stereoscopic omnidirectional sensor and the experimental environment

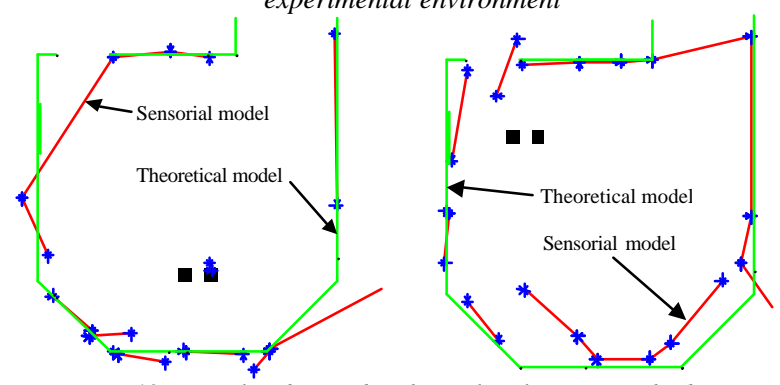

Figure 13 : Results obtained with our localization method on two stereoscopic acquisitions 
Figure 13 and Figure 14 presents the results of our localization method showing the final matching of the environment map with the stereoscopic sensorial model. On Figure 14 we can note that the dead reckoning position estimation is resetted for each acquisition with the absolute position estimation.

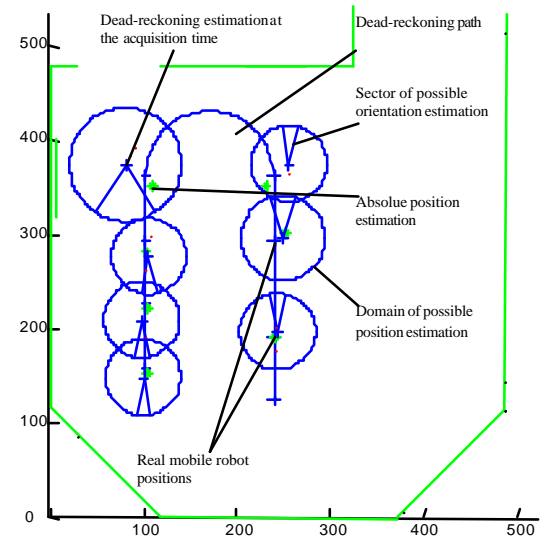

Figure 14 : Cooperation between the omnidirectional stereoscopic sensor and the dead-reckoning system.

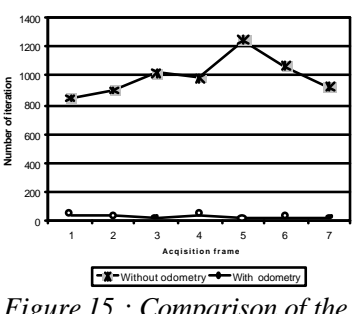

Figure $15 \cdot$ Comparison of the number of iteration with and without odometry.

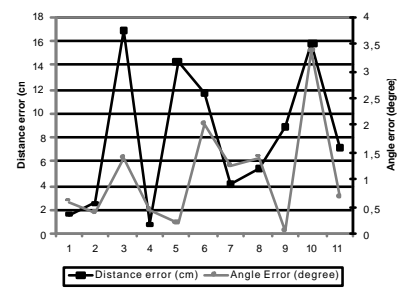

Figure 16 : Orientation and Cartesian error obtained on 11 absolute configuration estimation of the robot
In several acquisitions, we have quantify on Figure 15 the number of eliminated combinations with the deadreckoning in connection with the number with none use of odometry. We can note on the one hand that the preciseness of the localization method is great (error curve on Figure 16) and on the other hand that the error criterion for distance is highly discriminating since our method has given a correct matching for the totality of realized acquisitions. Moreover the reduction of the tested combinations with the dead-reckoning allows to obtain a robust absolute position estimation with an acceptable computation time.

\section{VIII.Conclusion}

We have developed a localization system based on a cooperation of a stereoscopic omnidirectional perception system and a dead-reckoning system. We have had to solve the main problem residing in the building of a robust sensorial model from two exteroceptive conical sensors. The optimization of the robustness is reached thanks to the information fusion from complementary and elementary treatments. We have used the fusion method from the Dempster-Shafer theory. It has allowed us to take into account the notion of weighting for each elementary treatment. We have tested our building method on several acquisitions in an indoor environment, which has allowed us to put into light the interest of our approach : whatever the type of environment, we reached an important precision on the sensorial considered primitives. We could thus develop a robust absolute localization algorithm by matching the stereoscopic sensorial model with the environment map. We have integrated to the matching stage a coherence position test linked to the deadreckoning estimation. This method allows to determine the robot's configuration with an important precision (a mean accuracy of $10 \mathrm{~cm}$ for the position) and mostly with an important robustness. The important number of significant data of the stereoscopic sensorial model got on an acquisition allows us to contemplate interesting prospects in connection with the environment incremental modelization in the case of an evolution in unknown environment.

\section{Acknowledgements}

This work was supported in part by "Région Picardie" under the project "Pôle DIVA".

\section{References}

[1] J. Crowley, "World modelling and position estimation for a mobile robot using ultrasonic ranging", Proc. of IEEE Conference on Robotics and Automation, Scottsdale, May 1989, p. 674-680.

[2] S. Atiya, G. Hager, "Real-time vision-based robot localization", IEEE Trans. On Robotics and Automation, Vol. $9 n^{\circ} 6$, pp. 785-800, December 1993.

[3] Y. Yagi, Y. Nishizawa, M. Yachida, "Map-based navigation for a mobile robot with omnidirectional image sensor COPIS", IEEE Trans. on Robotics and Automation Vol. 11, pp. 634-648, October 1995.

[4] J. Leonard, H. Durrant-Whyte, "Mobile robot localization by tracking geometric beacons.", IEEE Trans. on Robotics and Automation Vol. 7, pp. 89-97, June 1991.

[5] L. Delahoche, B. Marhic, C. Pégard, P. Vasseur "A navigation system based on an omnidirectional vision sensor", Proc. On IEEE/RSJ Int. Conf. on Intelligent Robots and Systems (IROS'97), France, September 1997.

[6] Durrant-Whyte, "The design of a radar-based Navigation System for large outdoor vehicles", Proc. IEEE Int. Conf. on Robotics and Automation, Aichi, Japan, pp. 764-769, May 1995.

[7] J. Gonzalez, A. Stenz, A. Ollero, "An iconic position estimator for a 2D laser rangefinder", IEEE Proc. of Int. Conf. on Robotics and Automation, Nice, pp 2646-2651, May 1992.

[8] G.A. SHAFER "A mathematical theory of evidence", Princeton : university press, 1976.

[9] R.O. Duda, P.E. Hart, "Pattern Classification and scene analysis", New York : Willey, 1973

[10] M. Wang, "Localisation Estimation and Analysis for Mobile Robots", In Autonomous Robot Vehicles, Cox I.J. et Wilfong, G.T. Editors, Springer Verlag, 1990. 